Path. Microbiol. 1970;36:I-VIII

\title{
Contents, Vol. 36, 1970
}

\section{Vol. 36,1970}

Pathologia et Microbiologia

Founded 1938 by A. v. Albertini, A. Grumbach and H. Mooser

Offizielles Organ der Schweiz. Mikrobiologischen Gesellschaft

Organe oíiiiciel de la Société Suisse de Microbiologie

Official Organ of the International Society of Geographical Pathology

Editors:

H. Ramseier, Zurich

J. R. Rüttner, Zurich

$\mathrm{s} \ll \mathrm{k}$

S. Karger · Basel · München · Paris · London · New York · Sydney

S. Karger AG, Arnold-Böcklin-Strasse 25, 4000 Basel 11 (Switzerland)

All rights, including those of translation into other languages, reserved. Photomechanical reproduction (photocopy, microcopy) of this volume or parts thereof without special permission of the publishers is prohibited.

(C) Copyright 1970 by S. Karger AG, Verlag für Medizin und Naturwissenschaften, Basel Printed in Switzerland by Friedrich Reinhardt AG, Basel Blocks: Aberegg-Steiner \& Co., Bern, and Steiner \& Co., Basel

Index

Ahmad-Zadeh, C. et Piguet, J. D.: Comparaison des poids moléculaires des chaînes polypeptidiques d'immunoglobulines de différentes origines, determines par électrophorèse en gel de polyacrylamide ... 305

Allet, B.: vide Piffaretti, J. Cl.

Anderson, D. L.: vide Tsien, H. C.

Bächi, T.: Elektronenmikroskopische Untersuchungen über den Infektionszyklus von Influenza A-Viren in Ehrlich-Aszites-Tumor-Zellen. - Electron

Microscopic Investigations on the Cycle of Infection of Influenza A Virus in Ehrlich Ascites Tumor Cells 81

Baniö, S. and Budic, Sonja: Efficiency of Medium B-2 for the Isolation of Tubercle Bacteria from Menstrual Blood and form Endometrium in

Female Genital Tuberculosis 123

Baumberger, U.: Growth of Vesicular Stomatitis Virus in Human Myeloblasts 161

Beneke, G.: vide Mohr, W.

Bertschinger, H. U.: Dissoziationsbedingte serologische Kreuzreaktionen bei Schweine-Enterotoxämie-Kolitypen. - Dissociation of E. coli Isolated from Pig Enterotoxemia Resulting in Serological Cross

Reactions 215,339

Bienz, K.: vide Löffler, $H$.

Bienz, K.: vide Weiss, Marianne 
Borman, J. B.: vide Laufer, A.

Budek, I.: vide Künzel, W.

Budic, Sonja: vide BaniC, S.

Büsser, E.; Pedio, G. und Rüttner, J. R.: Knochenläsionen und Metastasie-

rungsmuster in einem experimentellen Plasmozytom der BALB/c-

Maus (HIPA-Tumor). - Bone Lesions and Distribution of Metas-

tases in an Experimental Plasmacytom of BALB/c Mice (HIPA-

Tumor) 243

Davies, A. M.: vide Yarom, R.

Denis, Colette: vide Engelbrecht, E.

Dunkel, H.-J.: vide Mohr, W.

Eigenheer, F.: vide Löffler, $\mathrm{H}$.

Engelbrecht, E. et Denis, Colette: La reaction d'hémagglutination passive

pour la syphilis (antigène Reiter). Premiere partie: mise au point

technique. - The Indirect Hemagglutination Reaction in Syphilis.

Part I 44

Engelbrecht, E. et Denis, Colette: La reaction d'hémagglutination passive

pour la syphilis (antigène Reiter). Deuxième partie: La reaction face

aux tests habituels; etude de 50 serums. - The Indirect Hemag

glutination Reaction in Syphilis. Part II 51

VI

Index

Erill, S. und Gras, J.: A Serologic Method Using Charcoal Particles from Commercial India Ink. Application to Several Antigen-Antibody Systems with Special Reference to the Rheumatoid

Factor . . . 173

Ettlinger, L.: vide Krieg, P.

Friedman, I.: vide Laufer, A.

Garces, C. J.: vide Laufer, A.

Geisler, Ch.: vide Oberzill, W.

Germanier, R.: Selektion stark toxinogener Corynebacterium diphtheria-

Mutanten 286

Gonzenbach, P.: Wachstum und Ausbreitung des Ehrlich-Asziteskarzinoms der

Maus im Hühnerembryo. - Growth and Spread of Ehrlich Ascites

Carcinoma of the Mouse in the Chick Embryo 200

Gras, J.: vide Erill, S.

Gubler, H. U.: Über pigmentbildende Escherichien 344

Haller, O.: Replication of Influenza A Virus in Suspensions of Ehrlich Ascites

Tumor Cells Kept in vitro. Results Obtained by the Fluorescent

Antibody Technique

1

Jack, G. W.; Sykes, R. B. and Richmond, M. H.: The /?-Lactamases of Gram-

Negative Bacteria and their Transfer by R-Factors 289

Jacob, H. E.: Influence of Pretreatment of the Redoxpotential Electrode on the

Determination of Oxygen Pressure in Bacterial Cultures ... 57

Jacobi, H. D.: vide Künzel, W.

Katinger, H. und Meyrath, J.: Aspekte des Massentransfers bei der Verwer-

tung von Kohlenwasserstoffen durch Hefe 341 
Kaufhold, H. W.: vide Künzel, W.

Kellenberger, E. und Kellenberger-van der Kamp, C: Neue Ergebnisse

zur Formvererbung des Phagen T4 320

Kellenberger-van der Kamp, C: vide Kellenberger, E.

Korten, G.: vide Nimmich, W.

Krieg, P. and Ettlinger, L.: Kinetic Studies on Sorbose Fermentation . . 343

Künzel, W.; Jacobi, H. D.; Budek, I.; Kaufhold, H. W.; Meissner, Chr.

und Zschieschang, A.: Versuche zur quantitativen Erfassung von

Mykoplasmenwachstum. - Tests for the Quantitative Evaluation of

Mycoplasma Growth 27

Laufer, A.; Garces, C. L; Ron, N.; Friedman, I. and Borman, J. B.: Morphological and

Immunological Studies in Valvular Heart Surgery . . 230

Laufer, A.: vide Yarom, R.

Leonardopoulos, J. und Papavassiliou, J.: Eine Mikromethode zur Aus-

führung von Urease-Tests und PPA-Test. - Micromethod for the

Urease and PPA Tests 129

Link, F.; Schindler, M. und Majer, M.: Grippenschutzimpfung während

zweier Jahre in einem Industriebetrieb 281

Löffler, 1·L; Bienz, K; Schmid, U. und Eigenheer, F.: Verwendung des Elektronenmikroskopes bei der Diagnose von unklaren Virusinfek-tionen. Herpes-Virus-Enzephalitis und postvakzinale

Keratitis . . 324

Majer, M.: vide Link, F.

Meissner, Chr.: vide Künzel, W.

Index VII

Meyer, J.: vide Weiss, Marianne

Meyrath, J.: vide Katinger, $H$.

Mohr, W.; Beneke, G.; Dunkel, H.-J. und Murr, L.: Untersuchungen über die

Phagozytoseaktivität von Peritonealflüssigkeitszellen während der Neonatalperiode der Ratte. Investigations on the Phagocytic Activity of Rat Peritoneal Fluid Cells in the Neonatal Period . . . 153

Montoya, A.: Pale Cell Acanthoma (Degos) 38

Müller, H. E. und Werner, H.: In v/íro-Untersuchungen über das Vorkom-men von

Neuraminidase bei Bacteroides-Arten. - In vitro Studies in the Occurrence of Neuraminidase in

Bacteroides Species . . . 135

Murr, L.: vide Mohr, W.

Mussgay, M.: Gedanken zur Epidemiologie und Bekämpfung viraler Krank-

heiten unter Bedingungen der Massentierhaltung 325

Nimmich, W. und Korten, G.: Die chemische Zusammensetzung der Kleb-

síella-Lipopolysaccharide (O-Antigene). - The Chemical Composition

of Klebsiella Lipopolysaccharides (O-Antigens) 179

Novotný, P.: Über die Autolyse der Zellwände von Clostridium sordelli . . 285

Oberzill, W. und Geisler, Ch.: Einfluss methodischer Varianten auf die

Standardkurve bei Vitamin B12-Bestimmungen mit Lactobacillus

leichmannii. - Effect of Methodical Variations on the Standard

Curves in Vitamin B12-Determinations with Lactobacillus leich

mannii $\quad 193,340$ 
Papavassiliou, J. und Theodoratos, S.: Sensitivity of Salmonella paratyphi B to Colicines and Phase Variation 282

Papavassiliou, J.: vide Leonardopoulos, J.

Pardoe, Grace I.: The Inducible /j-Galactosidase (/1/8 \&gt; -Galactoside Galactohydrolase EC 3.2.1.23) of Klebsiella aerogenes NCIB 9479) ... 108

Pedio, G.: vide Büsser, E.

Piffaretti, J. Cl. et Allet, B.: Effet du chloramphenicol et de ses derives

acétylés sur la synthèse des protéines 303

Piguet, J. D.: vide Ahmad-Zadeh, C.

Pitton, J. S.: Contrôle génétique de la synthèse des pénicillinases chez quel-

ques bacilles Gram négatifs 299

Pitton, J. S.: vide Rassekh, M.

Raab, W. P.: Renal Effects of Antituberculous Antibiotics 73

Rassekh, M. et Pitton, J. S.: Distribution des modes de resistance à la streptomycine chez les souches sauvages d'entérobactéries 301

Reber, H.: Demonstrationen aus der klinischen Bakteriologie .... 279

Reilly, B. E.: vide Tsien, H. C.

Reuber, M. D.: Decreased Incidence of Thyroiditis in Aged Buffalo Strain

Rats Given 3-Methylcholanthrene 223

Richmond, M. H.: vide Jack, G. W.

Ron, N.: vide Laufer, A.

Rott, R.: Pathogenese von Viruskrankheiten 306

Rüttner, J. R.: vide Büsser, E.

Sackmann, W.: Dosierung und Wirkungsweise bakterizider Chemotherapeutika in Beziehung zur «Low Resistance Period» des Tierversuches . . 284

VIII

Index

Salfinger, M.: Zur Kinetik der Abtötung von Staphylococcus aureus durch

Formaldehyd 277

Schindler, M.: vide Link, F.

Sch $\gamma$ pper, E. und Steck, F.: Ätiologische Untersuchungen über Virusinfektionen in Kälber- und Rindermastbetrieben 338

Schmid, U.: vide Löffler, $H$.

Spicher, G.: Desinfektionsmittel und Desinfektionsverfahren, unter besonderer Berücksichtigung der Faktoren, die ihre Wirksamkeit und Brauchbar-

keit beeinflussen 259

Steck, F.: vide Schipper, E.

Sykes, R. B.: vide Jack, G. W.

Theodoratos, S.: vide Papavassiliou, J.

Tsien, H. C.; Reilly, B. E. and Anderson, D. L.: Rescue of Genetic Markers

from Bacteriophage 029 DNA Fragments 288

Weiss, Marianne; Meyer, J. und Bienz, K.: Elektronenmikroskopische Unter suchungen am Kern von Coxsackie-Virus-A9-infizierten Affennieren-

zellen 322

Werner, H.: vide Müller, H. E.

Yarom, R.; Laufer, A. and Davies, A. M.: Effect of Repeated Isoproterenol 
Administration in Young Rats. A Histological and Serological Study 65

Zschieschang, A.: vide Künzel, W.

Proceedings - Gesellschaftsberichte - Congrès Schweizerische Mikrobiologische Gesellschaft, 29. Jahresversammlung, 11. bis 13. Juni 1970 in Baden/AG. - Société Suisse de Microbiologie, 29e assemblée annuelle, les 11 au 13 juin 1970, à Baden/AG ... 259

Book Reviews 63,127,191, 256, 346

Subject Index 349 